\title{
Retroflexion, a costless endoscopic maneuver, increases adenoma detection rate in the ascending colon
}

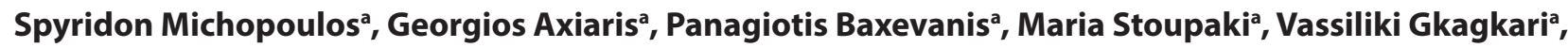 \\ Georgios Leonidakisa, Evanthia Zampelia, Maria Sotiropouloub ${ }^{b}$ Kalliopi Petrakic
}

Alexandra Hospital, Athens; Metropolitan Hospital, P. Faliro, Greece

\begin{abstract}
Background Missed polyps during colonoscopy are considered an important factor for interval cancer appearance, especially in the ascending colon (AC). We evaluated the contribution of retroflexion to polyp and adenoma detection in the AC.

Methods This prospective observational study included consecutive patients who underwent a complete colonoscopy between $06 / 2017$ and 06/2018. The AC was examined in 2 phases: the first included 2 forward views from the hepatic flexure to the cecum; the second involved a retroflexion in the cecum, inspection up to the hepatic flexure and reinsertion to the cecum.

Results The study included 655 patients, 628 (95.88\%) with successful retroflexion (mean age: $62.5 \pm 10.8$ years, 332 male). Indications for colonoscopy were screening in $33.28 \%$, follow up in $36.03 \%$, and diagnostic assessment in $30.69 \%$. In total, 286 polyps and 220 adenomas were detected in the AC. Phase 1 identified 119 adenomas, yielding an adenoma detection rate (ADR) in the AC of $14.2 \%$ (95\% confidence interval [CI] 11.52-16.84\%) while phase 2 identified 86 additional adenomas, improving the $\mathrm{ADR}$ in the $\mathrm{AC}$ to $22.75 \%$ (95\%CI 19.54-25.96\%; $\mathrm{P}<0.01)$. Adenoma miss rate was $39.1 \%(86 / 225)$ and per-patient adenoma miss rate was $11.15 \%$ (73/655). Retroflexion proved beneficial mainly in the upper third of the AC (odds ratio [OR] 4.29, 95\%CI 1.84-11.56; $\mathrm{P}<0.01)$ and for small $(<5 \mathrm{~mm})$ adenomas (OR 1.61, 95\%CI 1.02-2.56; $\mathrm{P}=0.04$ ). Multivariate analysis showed that age $>60$ years, detection of adenomas in forward views and the indication "follow up" influenced ADR during retroflexion.
\end{abstract}

Conclusion Retroflexion is a simple and safe maneuver that increases the ADR in the AC and should complete a second forward view.

Keywords Colonoscopy, retroflexion, adenoma detection rate, adenoma miss rate, polyp

Ann Gastroenterol 2020; 33 (6): 1-8

\section{Introduction}

Colorectal cancer (CRC) is an important health problem worldwide. Screening aims to diagnose premalignant

aGastroenterology Department, "Alexandra" Hospital, Athens (Spyridon Michopoulos, Georgios Axiaris, Panagiotis Baxevanis, Maria Stoupaki, Vassiliki Gkagkari, Georgios Leoniakis, Evanthia Zampeli); ${ }^{\text {Pathology }}$ Department, "Alexandra" Hospital, Athens (Maria Sotiropoulou); 'Pathology Department, "Metropolitan" Hospital, P. Faliro (Kalliopi Petraki), Greece

Conflict of Interest: None

Correspondence to: Spyridon Michopoulos, 6 Agias Kyriakis St., 14561 Athens, Greece, e-mail: michosp5@gmail.com

ClinicalTrials.gov ID number: NCT04086706

Received 21 July 2020; accepted 24 September 2020; published online 12 October 2020

DOI: https://doi.org/10.20524/aog.2020.0549 conditions (adenomas) or early stages of disease and it appears to have had a considerable impact on reducing CRC incidence and mortality [1]. Colonoscopy is a key tool in all CRC screening programs, either as the initial method or as a method to complement another positive screening test [2-6].

Most studies corroborate that colonoscopy contributes to the reduction of CRC mortality, more significantly in the distal rather than the proximal colon $[7,8]$. An increased rate of incomplete polypectomies or missed lesions in the proximal colon during colonoscopy could be an explanation for this observation in right-sided CRC $[9,10]$. In order to circumvent this problem, many approaches continue to be tested, such as improvement in the means of delivering contrast enhancers for colon chromoendoscopy, technological improvements in the endoscopes (high definition, optical magnification, digital chromoendoscopy, wide-angled lenses, etc.), or invention of new devices (endocap, endocuff, third eye, G-eye, etc.). Those techniques have a wide range of success for lesion detection, although they add a considerable cost $[11,12]$. 
On the other hand, simple maneuvers, such as change of patient position, water immersion or exchange colonoscopy, second forward-view examination or retroflexion in the ascending colon, may improve the adenoma detection rate (ADR) in the proximal colon [13-15]. Rectal retroflexion, although not universally accepted, has been suggested as an essential additional maneuver to better examine the lower rectum [16]. For a better inspection of the right colon, either a second forward view or retroflexion has been proposed but there is still uncertainty as to the best strategy. In a recent metaanalysis that included 4 studies with 1882 patients, when the second forward view was compared with retroflexion there was no difference in terms of adenoma miss rate (AMR) [17]. In our department, for many years, we have incorporated retroflexion in the ascending colon and rectum into the standard colonoscopy protocol.

The aim of our study was to examine the AMR when performing retroflexion systematically after a second forward view in the ascending colon and to evaluate the additional gain in terms of adenoma detection.

\section{Patients and methods}

We prospectively evaluated a cohort of consecutive patients for polyp detection in the ascending colon during complete colonoscopy in the "Alexandra" General Hospital, Athens, Greece, during a predetermined period (June 2017-June 2018). Inclusion criteria were as follows: patients older than 18 years, with a complete colonoscopy, with an indication for CRC screening, follow up or diagnostic assessment. In our study the 3 groups were defined as follows: a) screening group: asymptomatic patients aged $>50$ years undergoing colonoscopy for the first time; 2) follow-up group: patients who had undergone a previous colonoscopy (irrespective of the presence of polyps or not) in the context of CRC screening or post-polypectomy surveillance; and 3) symptomatic assessment group: patients undergoing colonoscopy for any symptomatic indication (transit abnormalities, blood per rectum, iron deficiency anemia, chronic diarrhea, etc.) requiring an endoscopic approach. Exclusion criteria precluded patients with a previous colectomy or abdominal surgery in the last 6 months; patients with polyposis syndromes or inflammatory bowel diseases; and cases unfit for polypectomy or where a polyp specimen was not retrieved for histology.

All colonoscopies were performed under continuous monitoring, with the patient under conscious sedation using midazolam and propofol provided by properly trained gastroenterologists or trainees who had a valid certification of advanced or basic life support (ALS or BLS). Olympus Evis Exera CF-H185L and 190L endoscopes were used with $\mathrm{CO}_{2}$ insufflation (OLYMPUS - UCR). An irrigation pump (OLYMPUS - OFP2) was used if needed, either for washing or for the water exchange technique. Split doses of oral sodium and potassium sulfate in combination (Eziclen ${ }^{\circledast}$ ) or PEG solutions (Klean Prep ${ }^{\circledast}$ or Fortrans ${ }^{\circledast}$ ) were used for bowel preparation, which was evaluated using the Segmental Boston Bowel
Preparation Scale (SBBS). Paris classification and narrow-band imaging (NBI) were used for the endoscopic characterization of the polyps before removal. The protocol of ascending colon examination encompassed 2 phases: an initial phase 1, divided into forward view 1a, which consisted of insertion from the right flexure to the cecum, followed by a second forward view $1 \mathrm{~b}$, namely withdrawal until the right flexure and reinsertion to the cecum; and a second phase (2, retroflexion) with a U-turn of the colonoscope in the cecum until the right flexure and then reverting to forward view and reinsertion to the cecum.

Concerning the endoscopists, 2 seniors and 4 trainees participated in the study. During all examinations of the right colon at least 2 observers were present, with the most experienced endoscopist (SM) always present, in order to reveal the genuine value of retroflexion by minimizing interobserver variability. Only 3 attempts to achieve retroflexion were permitted; a slight change of position from left lateral to decubitus was also permitted if needed. During the insertion and withdrawal phases of the forward and retroflexion views, polyps were mapped on a predetermined sketch of the right colon separated into 4 parts (cecum, lower, middle and upper third of the ascending colon, with the right flexure encompassed in the latter part) and were not removed until the end of the inspection. Only polyps of the ascending colon (lower, middle and upper third with the right flexure included) were included in our analysis, while cecal polyps were not.

Polypectomy followed, according to the previous mapping, and all polyps were collected and sent for histological examination. In addition to all tubular or tubulovillous adenomas, large serrated adenomas $(\geq 10 \mathrm{~mm})$, or those with dysplasia due to their malignant potential, were included in the group of "adenomas" for our analysis $[18,19]$. The remaining serrated or hyperplastic polyps were included in the group of "polyps". ADR was defined as the number of colonoscopies in which one or more adenomas was detected, divided by the total number of colonoscopies, and ADR in the ascending colon as the number of colonoscopies with at least one adenoma in the ascending colon, divided by the total number of colonoscopies. The AMR in the ascending colon was defined as the number of additional adenomas in the ascending colon detected by retroflexed view, divided by the total adenomas in the ascending colon detected with 2 forward views and the retroflexion. The per-patient miss rate was calculated as the number of patients with additional adenomas detected on retroflexion divided by the total number of patients who underwent the examination. Finally, we evaluated 2 additional quality parameters to assess the contribution of retroflexion to adenoma detection: adenomas per colonoscopy (APC), calculated by dividing the number of adenomas detected by the total number of colonoscopies, and adenomas per positive participant (APP), calculated by dividing the number of adenomas detected by the number of colonoscopies in which at least 1 adenoma was detected.

The study was conducted according to the ethical guidelines of the 1975 Declaration of Helsinki. The protocol of this noninterventional study, together with the informed consent document to be signed by the patients, were submitted and 
approved by the local ethics committee (Scientific Board of "Alexandra" Hospital).

\section{Statistical analysis}

In order to calculate our sample size, we hypothesized that the net benefit of retroflexion in terms of the ADR in the ascending colon would be at least $6 \%$ [17]. The sample size was calculated to be 652 patients for a power of 0.8 and a significance level of 0.05 . The applied technique was identical for all patients, therefore only 1 group of patients was required. We performed Intention to treat (ITT: failed retroflexion cases were included in the analysis) and per protocol (PP: successful retroflexion cases only) analyses. The location and size of each polyp were recorded, while its type was determined based on the histological report. Descriptive statistical analyses included computation of relative and absolute frequencies for categorical variables, or mean and standard deviation for continuous variables. We used the Exact McNemar's test for the ADR comparison in our main analysis. We performed stratified analysis considering the adenoma's position and size in the ascending colon, using the Exact McNemar's test. We investigated for factors associated with per-patient AMR using bivariate and multiple logistic regression analysis. P-values $<0.05$ were considered statistically significant and those between 0.05 and 0.10 were considered as indicative. All analyses were performed using Stata version 14.0 software.

\section{Results}

Six hundred seventy-seven (677) patients were initially included in the study; 22 patients were excluded (17 had a diagnosis of inflammatory bowel disease and 5 because there was no histology available for the resected polyps). Thus, 655 consecutive patients were included in our analysis. All had read and signed the informed consent form. All of them had a complete examination (cecal intubation 100\%) and were analyzed. The patients' mean age was $62.54 \pm 10.79$ years and 332 (50.69\%) were male. All had completed the first phase of the study ( 2 forward views in the ascent colon) while retroflexion was achieved in 628 (success rate for retroflexion 95.9\%). Their indication for colonoscopy was for screening in $33.28 \%$, followup colonoscopy in $36.03 \%$ and for diagnostic assessment related to symptoms or laboratory abnormalities in $30.69 \%$. Bowel preparation according to the Boston Bowel Preparation Scale was considered excellent (score=9) in 571 patients $(87.18 \%)$ (Table 1). No serious adverse events were observed and no hospitalization was needed after the procedures.

The ADR of the entire colon was 54.35\%, with APC 1.4 and APP 2.58, while for screening colonoscopies the ADR was $43.12 \%$ (53.19\% for males and $35.48 \%$ for females), with APC 0.98 and APP 2.28. When all patients were included (ITT analysis) ADR for the ascending colon was calculated at $22.75 \%$ (95\% confidence interval [CI] 19.54-25.96\%), leading to an added value for
Table 1 Descriptive characteristics for 655 patients undergoing colonoscopy

\begin{tabular}{lc}
\hline Variables & Value \\
\hline Age (years) & $62.54 \pm 10.79$ \\
Weight $(\mathrm{kg})$ & $76.5 \pm 15.16$ \\
Height $(\mathrm{cm})$ & $167.48 \pm 8.85$ \\
BMI $\left(\mathrm{kg} / \mathrm{m}^{2}\right)$ & $27.17 \pm 4.52$ \\
Colonoscope length $(\mathrm{cm})$ & $89.34 \pm 14.18$ \\
Sex & \\
Male & $332(50.69)$ \\
Female & $323(49.31)$ \\
Indication & \\
Screening & $218(33.28)$ \\
Follow up & $236(36.03)$ \\
Diagnostic & $201(30.69)$ \\
Boston cleansing index & \\
Poor & $5(0.76)$ \\
Moderate & $79(12.06)$ \\
Good & $571(87.18)$ \\
Retroflexion achievement & \\
Failure & $27(4.12)$ \\
1st attempt & $519(79.24)$ \\
2nd attempt & \\
3rd attempt & \\
\hline Values are $n(\%)$ and mean $\pm s d$ & \\
& \\
\hline
\end{tabular}

retroflexion of $8.55 \%$ (95\%CI 6.26-10.84\%; $\mathrm{P}<0.01)$. When only patients with successful retroflexion were analyzed (PP analysis), ADR for the ascending colon was 22.29\% (95\%CI 19.04-25.55\%), leading to an added value for retroflexion of $8.92 \%$ (95\%CI 6.5311.31\%; $\mathrm{P}<0.01$ ) (Table 2). The AMR was $39.1 \%(86 / 220)$ and the per-patient AMR 11.15\% (73/655) for the whole population (655 patients), while the rates were $41.95 \%(86 / 205)$ and $11.62 \%$ $(73 / 628)$, respectively, for those with successful retroflexion (628 patients). At least one adenoma was found in retroflexion in 56 patients without adenoma detection in either forward view, while in 17 patients with adenomas in forward views at least one additional adenoma was found in retroflexion. APC for the ascending colon was 0.34 and APP 1.48. Details of the distribution of polyps and adenomas identified in each phase in the ascending colon are shown in Table 3. Eighteen of 29 serrated polyps in the ascending colon were either large (10 polyps $\geq 10$ $\mathrm{mm}$ diameter) or had dysplasia $(\mathrm{n}=8)$ and were included in the adenomas group. In total, 220 adenomas were detected in the ascending colon, $119(60.91 \%)$ of them identified using a forward view. However, 86 (39.09\%) adenomas would have been missed without retroflexion, showing the important added value of this maneuver for $\mathrm{ADR}$ in the ascending colon $(\mathrm{P}<0.01)$. This was more pronounced in the upper third towards the right flexure, where $50 \%$ (95\%CI 35.68-64.32\%; $\mathrm{P}<0.01)$ of adenomas were detected only by retroflexion. Retroflexion was also found to be 
Table 2 Added value of retroflexion in the ascending colon

\begin{tabular}{llcccc}
\hline Variable & Protocol & Phase 1 & Phase 2 & Total & Added value \\
\hline N (PDR)(95\%CI) & ITT & $116(17.71)$ & $153(23.36)$ & $191(29.16)$ & $75(11.45)^{* * *}$ \\
& & $(14.79-20.63)$ & $(20.12-26.6)$ & $(25.68-32.64)$ & $(8.86-14.04)$ \\
& PP & $105(16.72)$ & $153(24.36)$ & $180(28.66)$ & $75(11.94)$ \\
& & $(13.8-19.64)$ & $(21-27.72)$ & $(25.13-32.2)$ & $(9.25-14.64)$ \\
N (ADR) (95\%CI) & ITT & $93(14.2)$ & 11417.4 & $149(22.75)$ & $56(8.55)^{* * *}$ \\
& & $(11.52-16.87)$ & $(14.5-20.31)$ & $(19.54-25.96)$ & $(6.26-10.84)$ \\
& PP & $84(13.38)$ & $114(18.15)$ & $140(22.29)$ & $56(8.92)^{* * *}$ \\
& & $(10.71-16.04)$ & $(15.14-21.17)$ & $(19.04-25.55)$ & $(6.53-11.31)$ \\
\hline
\end{tabular}



Phase 1: Two Forward views, Phase 2: Retroflexion, Total: Two Forward views + Retroflexion

ITT, Intention to treat analysis, 655 patients; PP, per protocol analysis, 628 patients, 27 without successful retroflexion were excluded

$N$, number of patients with at least one polyp/adenoma in the ascending colon; PDR, polyp detection rate; ADR, adenoma detection rate

Table 3 Distribution of polyps and adenomas in ascending colon identified in each phase, for 655 patients undergoing colonoscopy

\begin{tabular}{|c|c|c|c|c|c|c|c|c|c|c|}
\hline \multirow[t]{2}{*}{ No. } & \multicolumn{2}{|c|}{ Phase 1a } & \multicolumn{2}{|c|}{ Phase $1 \mathrm{~b}$} & \multicolumn{2}{|c|}{ Phase $1(a+b)$} & \multicolumn{2}{|c|}{ Phase 2} & \multicolumn{2}{|c|}{ Phase $1+2$} \\
\hline & Polyps & Adenomas & Polyps & Adenomas & Polyps & Adenomas & Polyps & Adenomas & Polyps & Adenomas \\
\hline 0 & 618 & 628 & 544 & 565 & 539 & 562 & 502 & 541 & 464 & 506 \\
\hline 1 & 32 & 22 & 76 & 61 & 81 & 64 & 110 & 83 & 127 & 103 \\
\hline 2 & 4 & 4 & 25 & 20 & 25 & 20 & 30 & 19 & 43 & 29 \\
\hline 3 & 1 & 1 & 7 & 7 & 7 & 7 & 9 & 9 & 14 & 11 \\
\hline 4 & 0 & 0 & 2 & 1 & 2 & 1 & 2 & 2 & 4 & 4 \\
\hline 5 & 0 & 0 & 1 & 1 & 1 & 1 & 2 & 1 & 3 & 2 \\
\hline Total & 43 & 33 & 160 & 131 & 165 & 134 & 215 & 161 & 286 & 220 \\
\hline
\end{tabular}

No, Number of polyps or adenomas; Phase 1a, $1^{\text {st }}$ Forward view; Phase 1b, $2^{\text {nd }}$ Forward view; Phase 1 (1a+1b), Phase 2 (Retroflexion); Phase 1+2 (2 forward views + retroflexion)

very effective in the detection of smaller adenomas. Detection of adenomas less than $5 \mathrm{~mm}$ was increased by $41.73 \%$ (95\%CI 32.37-51.1\%; $\mathrm{P}<0.01$ ) (Table 4). It should be noted that 11 polyps and 6 adenomas, all diminutive (diameter $<5 \mathrm{~mm}$ ), were found after the reversion of the colonoscope during the second phase. In addition, 10 polyps and 6 adenomas larger than $10 \mathrm{~mm}$ would have been missed if retroflexion had not been performed, while 4 adenomas $<10 \mathrm{~mm}$ had a tubulovillous histology. Furthermore, we examined if retroflexion was equally beneficial during the whole period of the study; by dividing the time into quartiles we found that retroflexion offered added value during the whole period of the study (Table 5). Cecal polyps were not incorporated in the analysis because they had been estimated only in forward views. We found 115 cecal adenomas in 93 patients (14.19\%), distributed according to the indication as follows: 27 of 218 (12.38\%) patients for screening, with no adenoma $>10 \mathrm{~mm} ; 34$ of $236(14.40 \%)$ patients in the follow-up group, with 3 adenomas $>10 \mathrm{~mm}$; and 32 of 201 (15.92\%) patients in the group with symptoms, with 9 adenomas $>10 \mathrm{~mm}$.

We performed univariate and multivariate analysis of the per-patient AMR in the ascending colon. Univariate analysis revealed significantly higher odds for people older than 60 years (odds ratio [OR] 3.04, 95\%CI 1.66-5.57; $\mathrm{P}<0.01$ ), adenoma detection during the forward views (OR 2.02,
95\%CI 1.12-3.66; $\mathrm{P}=0.02)$, and for the indications "follow up" (OR 2.45, 95\%CI 1.28-4.71; $\mathrm{P}<0.01$ ) and "symptoms" (OR 2.07, 95\% CI 1.04-4.11; $\mathrm{P}=0.04)$, compared to the indication "screening". Males were found to have indicatively higher odds than women (OR 1.55, 95\%CI 0.94-2.53; $\mathrm{P}=0.08$ ). Weight, height, body mass index, bowel preparation and colonoscope length at the cecum were not significant. In the multivariable model, age $>60$ years and the indication "follow up" were found to be statistically significant predictors for the perpatient AMR (Table 6).

\section{Discussion}

It is widely accepted that colonoscopy contributes less to the prevention of right colon cancer compared with the left $[7,8,20,21]$. The AMR is higher in the right colon and may contribute to the reduced efficacy [22]. Many techniques or devices have been proposed to counter this possible disadvantage of colonoscopy $[11,12,23]$. Retroflexion in the right colon is a well-described, easily learned, and (in experienced hands) safe procedure, without any additional cost. Serious adverse events are very rarely reported. In our 
Table 4 Details of 286 polyps and 220 adenomas identified in 655 patients (intention to treat analysis) and added value of retroflexion, stratified by polyp/adenoma size and position in the ascending colon

\begin{tabular}{|c|c|c|c|c|c|c|c|c|}
\hline \multirow[t]{2}{*}{ Details } & \multicolumn{2}{|c|}{ Two forward views } & \multicolumn{2}{|c|}{ Retroflexion } & \multicolumn{2}{|c|}{ Total } & \multicolumn{2}{|c|}{ Added value } \\
\hline & Polyps & Adenomas & Polyps & Adenomas & Polyps & Adenomas & Polyps & Adenomas \\
\hline \multicolumn{9}{|l|}{ Location } \\
\hline Bottom & $\begin{array}{c}40(66.6) \\
(54.74- \\
78.59)\end{array}$ & $\begin{array}{c}35(68.63) \\
(55.89- \\
81.36)\end{array}$ & $\begin{array}{c}36(60) \\
(47.6- \\
72.4)\end{array}$ & $\begin{array}{c}29(56.86) \\
(43.27- \\
70.46)\end{array}$ & $\begin{array}{c}60 \\
(100)\end{array}$ & $51(100)$ & $\begin{array}{c}20(33.33)^{* * *} \\
(19.74- \\
46.93)\end{array}$ & $\begin{array}{c}16(31.37)^{x+*} \\
(16.68- \\
46.07)\end{array}$ \\
\hline Middle & $\begin{array}{c}79(56.83) \\
(48.6- \\
65.07)\end{array}$ & $\begin{array}{c}69(63.3) \\
(54.25- \\
72.35)\end{array}$ & $\begin{array}{c}105(75.54) \\
(68.39- \\
82.69)\end{array}$ & $\begin{array}{c}79(49.07) \\
(64.09- \\
80.86)\end{array}$ & $139(100)$ & $109(100)$ & $\begin{array}{c}60(43.17)^{\star * *} \\
(34.21- \\
52.12)\end{array}$ & $\begin{array}{c}40(36.7)^{* * *} \\
(26.73- \\
46.66)\end{array}$ \\
\hline Upper & $\begin{array}{c}46(52.87) \\
(42.38- \\
63 . .36)\end{array}$ & $\begin{array}{l}30(50) \\
(37.35- \\
62.65)\end{array}$ & $\begin{array}{c}74(85.06) \\
(77.57- \\
92.54)\end{array}$ & $\begin{array}{c}53(88.33) \\
(80.21- \\
96.46)\end{array}$ & $\begin{array}{c}87 \\
(100)\end{array}$ & $60(100)$ & $\begin{array}{c}41(47.13)^{* * *} \\
(35.49- \\
58.77)\end{array}$ & $\begin{array}{c}30(50)^{x+*} \\
(35.68- \\
64.32)\end{array}$ \\
\hline \multicolumn{9}{|l|}{ Size } \\
\hline $0-4 \mathrm{~mm}$ & $\begin{array}{c}90(54.22) \\
(46.64- \\
61.8)\end{array}$ & $\begin{array}{c}74(58.27) \\
(49.69- \\
66.84)\end{array}$ & $\begin{array}{c}124(74.7) \\
(68.09- \\
81.31)\end{array}$ & $\begin{array}{c}94(74.02) \\
(66.39- \\
81.64)\end{array}$ & $166(100)$ & $127(100)$ & $\begin{array}{c}76(45.78)^{* * *} \\
(37.6- \\
53.96)\end{array}$ & $\begin{array}{c}53(41.73)^{n+*} \\
(32.37- \\
51.1)\end{array}$ \\
\hline $5-10 \mathrm{~mm}$ & $\begin{array}{c}50(58.82) \\
(48.36- \\
69.29)\end{array}$ & $\begin{array}{c}40(59.7) \\
(47.96- \\
82.43)\end{array}$ & $\begin{array}{c}65(76.47) \\
(67.45- \\
85.49)\end{array}$ & $\begin{array}{c}48(71.64) \\
(60.85- \\
82.43)\end{array}$ & $\begin{array}{c}85 \\
(100)\end{array}$ & $67(100)$ & $\begin{array}{c}35(41.18)^{* * *} \\
(29.54- \\
52.82)\end{array}$ & $\begin{array}{c}27(40.3)^{* * *} \\
(27.06- \\
53.54)\end{array}$ \\
\hline$>10 \mathrm{~mm}$ & $\begin{array}{c}25(71.43) \\
(56.46- \\
86.39)\end{array}$ & $\begin{array}{c}20(76.92) \\
(60.73- \\
93.12)\end{array}$ & $\begin{array}{c}26(74.29) \\
(59.8- \\
88.77)\end{array}$ & $\begin{array}{c}19(73.08) \\
(56.3- \\
90.13)\end{array}$ & $\begin{array}{c}35 \\
(100)\end{array}$ & $26(100)$ & $\begin{array}{c}10(28.57)^{* * *} \\
(10.75- \\
46.39)\end{array}$ & $\begin{array}{c}6(23.08)^{* *} \\
(3.04- \\
43.12)\end{array}$ \\
\hline Total & $\begin{array}{c}165(57.69) \\
(51.97- \\
63.42)\end{array}$ & $\begin{array}{c}134(60.91) \\
(54.46- \\
67.36)\end{array}$ & $\begin{array}{c}215(75.17) \\
(70.17- \\
80.18)\end{array}$ & $\begin{array}{c}161(73.18) \\
(67.33- \\
79.04)\end{array}$ & $\begin{array}{c}286 \\
(100)\end{array}$ & $220(100)$ & $\begin{array}{c}121(42.31) \\
* * * \\
(36.23- \\
48.38)\end{array}$ & $\begin{array}{c}86(39.09)^{n+*} \\
(32.19- \\
45.99)\end{array}$ \\
\hline
\end{tabular}

Values are $\mathrm{n}(\%),\left(95 \%\right.$ confidence interval), ${ }^{*} \mathrm{P}$-value $<0.1,{ }^{* *} \mathrm{P}$-value $<0.05,{ }^{, * *} \mathrm{P}$-value $<0.01$

Table 5 Added value of retroflexion view in terms of ADR during the 4 trimesters of the study, for 655 patients. June 2017-June 2018

\begin{tabular}{lcccc}
\hline ADR\% $(95 \% \mathrm{CI})$ & $1^{\text {st }}$ trimester & $2^{\text {nd }}$ trimester & $3^{\text {rd }}$ trimester & $4^{\text {th }}$ trimester \\
\hline Phase 1 & $16.67(10.92-22.41)$ & $12.96(7.42-18.14)$ & $15.95(10.34-21.57)$ & $11.31(6.52-16.1)$ \\
Total (phase1 +2) & $26.54(19.74-33.34)$ & $20.99(14.72-27.26)$ & $25.15(18.49-31.81)$ & $18.45(12.59-24.32)$ \\
Difference (Total - phase1) & $9.87(4.67-15.09)$ & $8.02(3.22-12.83)$ & $9.2(4.15-14.25)$ & $7.14(2.65-11.63)$ \\
& $\mathrm{P}<0.01$ & $\mathrm{P}<0.01$ & $\mathrm{P}<0.01$ & $\mathrm{P}<0.01$ \\
\hline
\end{tabular}

Phase 1: Two Forward views, Phase 2: Retroflexion, Total: 2 Forward views + retroflexion

$C I$, confidence interval, $A D R$, adenoma detection rate

study, the success rate of retroflexion was $95.9 \%$ with no serious adverse events, corroborating the results of most previous studies aiming to evaluate the safety and feasibility of retroflexion in the ascending colon [24-32].

In 2 studies $[27,29]$, retroflexion was found to be beneficial when compared to a meticulous single-standard forward view during withdrawal. A randomized controlled trial showed similar benefits for forward view or retroflexion [30]. A meta-analysis comparing colonoscopy including right colon retroflexion with conventional colonoscopy found an AMR of $16.9 \%$, with a range of $4.7-33.3 \%$, supporting the usefulness of retroflexion [15]. In a recent meta-analysis a second forward view was equally effective as retroflexion in increasing ADR in the right colon [17]. Our results concerning the efficacy of retroflexion in improving the polyp and ADR as well as AMR are the highest in the literature. Many parameters could explain those findings. Our study was performed using high-quality, high-definition (HD) endoscopes, which may have contributed to the high rate of polyps revealed by retroflexion, especially for small or medium size polyps, as was recently described for HD endoscopes [31,32].

Three studies have many similarities with ours, but with the main methodological difference that they removed polyps upon detection, and not after the completion of the inspection as we did $[25,28,33]$. Triantafyllou et al did not find a benefit from additional retroflexion, but they used non-HD endoscopes [25]. Lee et al [33] and Miyamoto et al [28] found that retroflexion following 2 forward-withdrawal examinations was beneficial 
Table 6 Multivariate analysis for per-patient adenoma miss rate in 655 patients

\begin{tabular}{|c|c|c|c|}
\hline \multirow[t]{2}{*}{ Characteristic } & \multicolumn{2}{|c|}{ Multivariate analysis } & \multirow[t]{2}{*}{ P-value } \\
\hline & OR & $95 \% \mathrm{CI}$ & \\
\hline \multicolumn{4}{|l|}{ Age (years) } \\
\hline$<60$ & Reference & & \\
\hline$\geq 60$ & 2.7 & $1.46-4.98$ & $0.01^{* * *}$ \\
\hline \multicolumn{4}{|c|}{ Adenomas in forward } \\
\hline No & Reference & & \\
\hline Yes & 1.78 & $0.97-3.27$ & $0.06^{*}$ \\
\hline \multicolumn{4}{|l|}{ Indication } \\
\hline Screening & Reference & & \\
\hline Follow up & 2.14 & $1.11-4.15$ & $0.02^{* *}$ \\
\hline Symptoms & 1.84 & $0.92-3.69$ & $0.09^{*}$ \\
\hline
\end{tabular}

mainly near the hepatic flexure. However, AMR and per-patient AMR were lower in these 2 studies compared with ours. In the case of Lee et al [33], this can be partially explained because the success rate of retroflexion was $82.4 \%$ in their study, while in ours it was $95.9 \%$. In another similar study published only in abstract form, $30 \%$ of the ascending colon polyps were detected by retroflexion, although the success rate in that study was only $76.6 \%$ [34]. Most of the additional polyps found in our study by retroflexion were towards the right flexure and were of small or medium size. Retroflexion performed less well at the proximal part of the ascending colon, supporting our point of view that it should be considered as a complementary rather than an alternative maneuver to forward view (Table 3). The main differences between our study and the very similar studies of Lee et al [33] and Miyamoto et al [28] are: 1) they removed the polyps upon inspection, while we did so only after finishing the mapping in both views; 2) they examined the colon only during withdrawal, while we also tried to meticulously examine it during the 2 insertions; and 3) Lee et al incorporated cecal polyps in their analysis, while Miyamoto et al refer to the right colon probably incorporating cecal polyps, although they are not clearly mentioned. Cecal polyps were not incorporated in our analysis but only polyps in the ascending colon, distal to the ileocecal valve. This was deliberate, because all cecal polyps were detected during the forward views and retroflexion was not efficient in detecting them. Other studies either do not specify or include cecal polyps in their analysis, thus diminishing the value of retroflexion. In our analysis we intentionally included the 11 polyps and 6 adenomas found after colonoscope reversion in the retroflexion phase, because this act would not be performed without retroflexion.

Inspection time during withdrawal is important; it was shown that when withdrawal time in the right colon was $\geq 2$ min the ADR, PDR, serrated polyp detection rate and APC were significantly higher [35]. In a subgroup analysis of 400 patients, the net inspection time in our study was
$65.89 \pm 19.28 \mathrm{sec}$ for phase 1 ( 2 forward views) and $58.95 \pm 15.57$ sec for phase 2 (retroflexion) showing that the total inspection time was $>2$ min. In all previous studies, polyps detected in forward view were resected prior to performing retroflexion, while in our study all polyps were mapped meticulously to avoid double counting and were resected after the whole procedure of inspection, including retroflexion, had been completed. Polyp resection prior to retroflexion could increase the inspection time of the forward view by increasing the time of the inspection of the surrounding area during the time of polypectomy procedure. Moreover, the use of dyes or even the presence of blood after polypectomy may change the endoscopic field, influencing the inspection.

The presence of additional qualified observers during colonoscopy increases polyp detection, especially for those with low to moderate ADR [36,37]. Furthermore, trainee ADR is higher under a high ADR supervisor [38]. In our study, at least 2 observers were present, with the most experienced endoscopist (SM) always present, in order to determine the genuine value of retroflexion by minimizing the interobserver variability. Consequently, the individual ADR in this study cannot be separately evaluated for the trainees and the senior endoscopists. Instead, this may have contributed to the increased number of polyps detected, although this was true not only for retroflexion but also for the 2 forward views.

In our study the ADR was high. The same annotation can be made for our patients' excellent bowel preparation, contributing to the high ADR. In addition, we evaluated the ADR for screening colonoscopies separately (43.12\%; 53.19\% for males and $35.48 \%$ for females) but we additionally utilized the overall ADR (54.35\%) to better unveil the usefulness of retroflexion [39]. Retroflexion was equally beneficial during the 4 trimesters of our study, indicating that the added value of retroflexion was maintained during the entire study period (Table 5).

Advanced polyps were detected in 25 patients in forward view and in an additional 10 during retroflexion. Moreover, we would like to point out that several times during the polypectomy procedure we were unable, despite many attempts, to visualize in forward view polyps detected during retroflexion. In a recent analysis of 8 randomized trials it was found that a right-side located adenoma is not more frequently missed than a left-side located one [40]. Our study was not designed for comparison between right- and left-sided AMR. However, as the authors of the above analysis commented, inherent differences between trials cannot be excluded and, although right-sided lesions were not more frequently missed under study conditions, quality measures should focus on right sided (and flat) colonic lesions. In a recent meta-analysis, Aziz et al [41] found no significant difference in the proximal ADR (in the right colon) between the use of either distal attachments (endocuff, endocap) or electronic chromoendoscopy compared with the normal HD endoscopes. Most additional polyps detected with retroflexion in our study were small; likewise, in a randomized study comparing the use of computer-aided detection of adenomas vs. conventional colonoscopy, the ADR increase with artificial intelligence was mainly due to diminutive adenomas with less contribution to the CRC risk [42]. 
The non-randomization between the 2 phases of inspection could be considered a limitation of our study. However, retroflexion was deliberately always performed as the second examination and not randomly, because the aim of our study was not to compare retroflexion with a second forward view, but to reveal the eventual additional value of retroflexion in an everyday clinical setting.

It is widely accepted that there is a great heterogeneity in everyday endoscopic practice and that many endoscopists utilize neither a second forward view nor retroflexion, but instead only a single forward view for examining the ascending colon. According to our results, retroflexion should be used not as an alternative, but as an additional maneuver. Its added value was revealed in our study, where many favorable parameters coexisted: HD endoscopes, NBI, good sedation, excellent cleaning of the bowel, high expertise and more than 2 observers during the procedure. This practice may increase the quality of colonoscopy in the right colon provided that both steps are accompanied by a watchful inspection of the examined area.

\section{Summary Box}

\section{What is already known:}

- Interval colorectal cancers are more frequently encountered in the right colon

- A possible explanation could be suboptimal polyp detection in the ascending colon

- It is still controversial whether or not retroflexion performs better than a second forward view for polyp detection in the ascending colon

\section{What the new findings are:}

- Retroflexion in addition to 2 forward views in the ascending colon adds substantially to the adenoma detection rate

- Most of the additional polyps were found towards the right flexure

- Retroflexion performed less well in the proximal part of the ascending colon and should be considered as a complementary rather than an alternative maneuver to forward views

\section{References}

1. Zauber AG, Lansdorp-Vogelaar I, Knudsen AB, Wilschut J, van Ballegooijen M, Kuntz KM. Evaluating test strategies for colorectal cancer screening: a decision analysis for the U.S. Preventive Services Task Force. Ann Intern Med 2008;149:659-669.

2. Fedewa SA, Goodman M, Flanders WD, et al. Elimination of costsharing and receipt of screening for colorectal and breast cancer. Cancer 2015;121:3272-3280.

3. Schenck AP, Peacock SC, Klabunde CN, Lapin P, Coan JF,
Brown ML. Trends in colorectal cancer test use in the medicare population, 1998-2005. Am J Prev Med 2009;37:1-7.

4. Kaminski MF, Kraszewska E, Rupinski M, Laskowska M, Wieszczy P, Regula J. Design of the Polish Colonoscopy Screening Program: a randomized health services study. Endoscopy 2015;47:1144-1150.

5. Canadian Task Force on Preventive Health Care. Recommendations on screening for colorectal cancer in primary care. CMAJ 2016;188:340-348.

6. Brenner H, Chang-Claude J, Seiler CM, Rickert A, Hoffmeister M. Protection from colorectal cancer after colonoscopy: a populationbased, case-control study. Ann Intern Med 2011;154:22-30.

7. Rex DK, Boland CR, Dominitz JA, et al. Colorectal cancer screening: recommendations for physicians and patients from the U.S. Multi-Society Task Force on Colorectal Cancer. Am J Gastroenterol 2017;112:1016-1030.

8. Doubeni CA, Corley DA, Quinn VP, et al. Effectiveness of screening colonoscopy in reducing the risk of death from right and left colon cancer: a large community-based study. Gut 2018;67:291-298.

9. Samadder NJ, Curtin K, Tuohy TM, et al. Characteristics of missed or interval colorectal cancer and patient survival: a populationbased study. Gastroenterology 2014;146:950-960.

10. Lee J, Park SW, Kim YS, et al. Risk factors of missed colorectal lesions after colonoscopy. Medicine (Baltimore) 2017;96:e7468.

11. Facciorusso A, Triantafyllou K, Murad MH, et al. Compared abilities of endoscopic techniques to increase colon adenoma detection rates: a network meta-analysis. Clin Gastroenterol Hepatol 2019;17:2439-2454.

12. Repici A, Wallace MB, East JE, et al. Efficacy of per-oral methylene blue formulation for screening colonoscopy. Gastroenterology 2019;156:2198-2207.

13. East JE, Bassett P, Arebi N, Thomas-Gibson S, Guenther T, Saunders BP. Dynamic patient position changes during colonoscope withdrawal increase adenoma detection: a randomized, crossover trial. Gastrointest Endosc 2011;73:456-463.

14. Clark BT, Parikh ND, Laine L. Yield of repeat forward-view examination of the right side of the colon in screening and surveillance colonoscopy. Gastrointest Endosc 2016;84:126-132.

15. Cohen J, Grunwald D, Grossberg LB, Sawhney MS. The effect of right colon retroflexion on adenoma detection: a systematic review and meta-analysis. J Clin Gastroenterol 2017;51:818-824.

16. Rees CJ, Thomas Gibson S, Rutter MD, et al; British Society of Gastroenterology, the Joint Advisory Group on GI Endoscopy, the Association of Coloproctology of Great Britain and Ireland. UK key performance indicators and quality assurance standards for colonoscopy. Gut 2016;65:1923-1929.

17. Desai M, Bilal M, Hamade N, et al. Increasing adenoma detection rates in the right side of the colon comparing retroflexion with a second forward view: a systematic review. Gastrointest Endosc 2019;89:453-459.

18. Lieberman DA, Rex DK, Winawer SJ, Giardiello FM, Johnson DA, Levin TR. Guidelines for colonoscopy surveillance after screening and polypectomy: a consensus update by the US Multi-Society Task Force on Colorectal Cancer. Gastroenterology 2012;143:844-857.

19. East JE, Atkin WS, Bateman AC, et al. British Society of Gastroenterology position statement on serrated polyps in the colon and rectum. Gut 2017;66:1181-1196.

20. Brenner H, Hoffmeister M, Arndt V, Stegmaier C, Altenhofen L, Haug U. Protection from right- and left-sided colorectal neoplasms after colonoscopy: population-based study. I Natl Cancer Inst 2010;102:89-95.

21. Singh H, Nugent Z, Demers AA, Kliewer EV, Mahmud SM, Bernstein CN. The reduction in colorectal cancer mortality after colonoscopy varies by site of the cancer. Gastroenterology 2010;139:1128-1137. 
22. Xiang L, Zhan Q, Zhao XH, et al. Risk factors associated with missed colorectal flat adenoma: a multicenter retrospective tandem colonoscopy study. World J Gastroenterol 2014;20:10927-10937.

23. Jain D, Sandhu N, Singhal S. New developments in mechanical enhancement of colonoscopy: cuffs, caps and rings. Digestion 2016;93:234-247.

24. Harrison M, Singh N, Rex DK. Impact of proximal colon retroflexion on adenoma miss rates. Am J Gastroenterol 2004;99:519-522.

25. Triantafyllou K, Tziatzios G, Sioulas AD, et al. Diagnostic yield of scope retroflexion in the right colon: A prospective cohort study. Dig Liver Dis 2016;48:176-181.

26. Kim HU, Boo SJ, Na SY, Song HJ. [Additional polyp detection rate using colonoscopic retroflexion in right colon]. Korean $J$ Gastroenterol 2015;65:90-98.

27. Chandran S, Parker F, Vaughan R, et al. Right-sided adenoma detection with retroflexion versus forward-view colonoscopy. Gastrointest Endosc 2015;81:608-613.

28. Miyamoto H, Naoe H, Oda Y, et al. Impact of retroflexion in the right colon after repeated forward-view examinations. JGH Open 2018;2:282-287.

29. Hewett DG, Rex DK. Miss rate of right-sided colon examination during colonoscopy defined by retroflexion: an observational study. Gastrointest Endosc 2011;74:246-252.

30. Kushnir VM, Oh YS, Hollander T, et al. Impact of retroflexion vs. second forward view examination of the right colon on adenoma detection: a comparison study. Am J Gastroenterol 2015;110:415-422.

31. Jrebi NY, Hefty M, Jalouta T, et al. High-definition colonoscopy increases adenoma detection rate. Surg Endosc 2017;31:78-84.

32. Bond A, O’Toole P, Fisher G, et al. New-generation high-definition colonoscopes increase adenoma detection when screening a moderate-risk population for colorectal cancer. Clin Colorectal Cancer 2017;16:44-50.

33. Lee HS, Jeon SW, Park HY, Yeo SJ. Improved detection of right colon adenomas with additional retroflexion following two forward-view examinations: a prospective study. Endoscopy 2017;49:334-341.

34. Vargas A, Alburquerque M, Figa M, González-Huix F. Sa1642 Ascending colon exploration by retroviewing: technical feasibility and diagnosis performance. Gastrointest Endosc 2015;81:AB293.

35. Jung Y, Joo YE, Kim HG, et al. Relationship between the endoscopic withdrawal time and adenoma/polyp detection rate in individual colonic segments: a KASID multicenter study. Gastrointest Endosc 2019;89:523-530.

36. Lee CK, Park DI, Lee SH, et al. Participation by experienced endoscopy nurses increases the detection rate of colon polyps during a screening colonoscopy: a multicenter, prospective, randomized study. Gastrointest Endosc 2011;74:1094-1102.

37. Tziatzios G, Gkolfakis P, Triantafyllou K. Effect of fellow involvement on colonoscopy outcomes: A systematic review and meta-analysis. Dig Liver Dis 2019;51:1079-1085.

38. Mahadev S, Jin Z, Lebwohl B, et al. Trainee colonoscopy quality is influenced by the independent and unobserved performance characteristics of supervising physicians. Endosc Int Open 2019;7:E74-E82.

39. Rex DK, Ponugoti PL. Calculating the adenoma detection rate in screening colonoscopies only: Is it necessary? Can it be gamed? Endoscopy 2017;49:1069-1074.

40. Zimmermann-Fraedrich K, Sehner S, Rex DK, et al. Right-sided location not associated with missed colorectal adenomas in an individual-level reanalysis of tandem colonoscopy studies. Gastroenterology 2019;157:660-671.

41. Aziz M, Desai M, Hassan S, et al. Improving serrated adenoma detection rate in the colon by electronic chromoendoscopy and distal attachment: systematic review and meta-analysis. Gastrointest Endosc 2019;90:721-731.

42. Wang P, Berzin TM, Glissen Brown JR, et al. Real-time automatic detection system increases colonoscopic polyp and adenoma detection rates: a prospective randomised controlled study. Gut 2019;68:1813-1819. 